\title{
Blockchain and Supply Chain "Double-Chain Integration” Development: Research Review and Outlook
}

\author{
Feng Wei \\ GuangXi University of Science and Technology \\ LiuZhou, China
}

\author{
Shanshan Song \\ School of Economics and Management \\ GuangXi University of Science and Technology \\ LiuZhou, China \\ shansong0212@163.com
}

\begin{abstract}
The de-centered, de-trusted, and traceable technical advantages of blockchain can be used to solve the problem of lack of trust and product tracking difficulties in multi-agent transactions in the supply chain. Thus, the supply chain has become a natural use of blockchain technology. The integration of chain and supply chain development can solve the problem of resource cross-fusion configuration between different parallel industry chains. At present, the research has only prospected the application of blockchain technology in a specific industry supply chain, and lacks a comprehensive systematic review and induction of the development of "double-chain integration". Considering the practical importance of the research topic of blockchain and supply chain integration development, this paper systematically sorts out and summarizes the related literatures of "double-chain fusion" development, and further constructs the research framework of supply chain intelligent governance.Block chain technology provides a new direction for the construction of supply chain informatization, which is the basis of realizing intelligent management of supply chain. Based on this, the research in this paper provides theoretical support for further research and practical operation of "double-chain fusion".
\end{abstract}

Keywords_blockchain; supplychain; research review; future prospects

\section{INTRODUCTION}

During the two sessions in 2019, 15 provincial and municipal proposals related to blockchain technology were included in the government work report. In 2018, well-known large companies at home and abroad participated in blockchain projects, enriching the practical application of blockchain technology. In 2018, there were 3732 search papers on cnn.com with blockchain as the key word. Compared with 1586 papers in 2017, the research on blockchain has been expanded in both quantity and content. It can be seen that the current research on blockchain has received attention from the political, industrial and academic levels. The blockchain is an

Innovation Project of GuangXi University of Science and Technology Graduate Education "Evaluation of Guangxi Automobile Supply Chain Information Utilization Efficiency from the Perspective of Information Ecology” (GKY201912)

Guangxi Education Science '13th Five-Year' Planning Project "Innovative Research on the Deep Integration Mechanism of the Application of Undergraduate Higher Education in Guangxi” (2017A037)

Guangxi Education Science ‘13th Five-Year’ Plan 2017 Guangxi Private Education Research Project "Research on the Development of High Quality Characteristics of Guangxi Private Colleges and Universities” (2017ZMB004)
Internet-based distributed ledger technology and a decentralized database[1].Because the blockchain has the characteristics of decentralization, trustless, traceability, and non-tampering, it can break the information island and improve the quality, stock and credibility of the data. In contrast, the supply chain has multiple trust entities, multi-party cooperation, and the characteristics of low-frequency trading, complete business logic, and non-centralized upstream and downstream organizational differences and the industrial chain ecology that can be closed upstream and downstream are the natural use of blockchain. Liu Dacheng (2016) of Tsinghua University introduced the concept of blockchain into the field of logistics supply chain, and first proposed the concept of "double-chain fusion" between blockchain and the supply chain. "Doublechain fusion" refers to making full use of the two unique advantages of the blockchain to make up for the two shortcomings of the supply chain; using the two advantages of the supply chain to solve the two pain points of the blockchain. It aims to break through the bottleneck of traditional supply chain management problems by integrating and real-time reflecting the real dynamics of all links in the supply chain through blockchain technology, sharing account books, opening up data islands, enhancing product anti-counterfeiting and traceability, and ensuring product quality. Therefore, it is important to introduce blockchain into supply chain management.

\section{A SUMMARY OF THE RESEARCH STATUS OF "DOUBLE CHAIN FUSION "DEVELOPMENT}

The arrival of the era of personalized production has led to an accelerated rate of information output and renewal in the supply chain. The information quality problems such as information distortion and information islands are becoming more and more serious. And the reduction in the quality of information will exacerbate the crisis of trust among members of the supply chain. Trust is the foundation for establishing supply chain partnership. In the past, the establishment of trust was inseparable from formal contract mechanism and informal relationship mechanism. However, with the progress of the society and the development of science and technology, information technology represented by blockchain provides a new means for the establishment of trust between supply chain partners [2]. Blockchain is by far the first technical means to 
solve the credit problem. It uses the strict data authenticity verification mechanism to ensure the credit certificate of social activities and trading activities, which fundamentally creates the possibility of "double-chain integration" development. This paper discusses the research status of "double-chain" fusion development from four aspects: "double-chain" fusion development theoretical model research, application scenario research, technical architecture research, and practical application research.

\section{A. Research on the Theory Model of "Double Chain" Fusion Development}

Block chain-based B2B+B2C supply chain principal transaction structure and dynamic multi-center collaborative authentication model use the trust proof mechanism of block chain which does not need credit data to solve the problem of difficult authentication of trading behaviors between trading entities in the supply chain (Zhu Jianming and $\mathrm{Fu}$ Yonggui, 2016) [3], so as to ensure high stability of the supply chain.The product information traceability and the anti-counterfeiting model of B2C e-commerce platform are focused on solving the information asymmetry, product information traceability and anti-counterfeiting problems in B2C sales(Ding Qingyang and Zhu Jianming, 2017). First ,using the Internet of Things technology to ensure that the information uploaded to the blockchain is complete and true availability, and then use blockchain technology to solve the problem of information traceability in the process of information collection, circulation, sharing, etc. The combination of Internet of Things and blockchain technology is beneficial to the management of supply chain logistics information resources. The ecological model of supply chain logistics information has the characteristics of anti-aggression, data confidentiality, selfrepair and operational ecology to ensure the ecological harmony of logistics supply chain information (Wang Chuanlei, 2017) [4]. The supply chain prototype system further improves the level of supply chain informatization by solving the trust problem between supply chain subjects, regulatory traceability problem, data privacy protection problem and the automation of information input, reducing human error (Xie Xiaorong, 2017). Most of the theoretical models built around the development of "double-chain" integration are cut from the supply chain information resource management. The aim is to realize the real traceability of information in the supply chain, ensure the effective transaction, and reduce the potential risks such as supply chain opportunism through the characteristics of the blockchain technology, thereby further improving the efficiency of the supply chain.

\section{B. "Double-chain" fusion application scenario research}

The supply chain finance itself has financial attributes and strong digital characteristics. Compared with traditional supply chain business, it is easier to use block chain technology. In the field of supply chain finance, it is difficult and expensive for SEMs to obtain financing due to their reliance on core enterprises for the credit extension and the difficulty in transferring credit information. Specifically, it is difficult to verify the authenticity of trade background of members in the supply chain, which makes the validity of supply chain platform data difficult to verify. The nature of the centralized structure of the supply chain financial system determines that when multiple systems are connected, there are security risks and low efficiency pain points. The "technical trust" based on blockchain can solve the trust problem through technical means, which can help small and medium-sized enterprises to finance and solve the problem of difficult separation and circulation of bills, so as to improve the efficiency of supply chain finance. By reducing the cost and complexity of the supply chain financial information system expansion, the security of the supply chain financial platform is enhanced.

Food and drug safety have always been a key concern for people's livelihood. The Changchun Changsheng rabies vaccine production record fraud incident once again sounded the alarm for food and drug safety. "Maintaining the safety of the people's tongue" has become one of the hot topics of the NPC and PCC in 2019.Agricultural products supply chain are the key regulatory field of food safety issues, so blockchain technology can be introduced into agricultural products supply chain. The advantages of blockchain technology (decentralized, distributed storage, open and transparent) can be used to solve the problems of the agricultural supply chain users' lack of trust, the difficulty in ensuring the information security of agricultural products, and the difficulty in tracing the quality of agricultural products. For example, blockchain-based agricultural product logic architecture can provide financial services and information sharing between upstream and downstream enterprises, and can also protect information security and privacy protection, breaking technical barriers in traditional supply chains (Yu Lina, 2017)[5]; through the integration of food traceability key standards and technologies, the food safety traceability system architecture of the Internet of Things plus blockchain will be established, and the traceability system will be used to realize the transparent management of supply, warehousing and distribution to the final retail end. Some scholars have further embodied it, and applied blockchain technology to the field of cold chain logistics of agricultural products. The agricultural product supply chain solution based on blockchain technology strengthens the traceability, reliability, anti-counterfeiting and information timeliness of agricultural products, improves the efficiency of supply chain, and provides an important way to realize the value-added of modern agricultural product supply chain.

In addition to researching the agricultural product supply chain, domestic and foreign scholars also conduct research on specific supply chains such as the pharmaceutical supply chain, the cigarette supply chain, and the manufacturing supply chain. Rerjers and Coeckelbergh's research shows that the drug inspection information based on blockchain technology itself makes the information more stable and reliable due to distributed storage, which can improve the inspection speed of the drug supply chain[6]. It is used to solve the core problems of high drug prices, information blocking, and traceability difficulties in the drug supply chain system. Due to the high cost, low efficiency, high risk and other core issues of the cigarette supply chain and the manufacturing supply chain system, the introduction of blockchain technology can ensure the transparency and traceability of information, thereby reducing the cost and operation of supply chain management risk[7] . 


\section{Research on "Double Chain" Fusion Technology Architecture}

The characteristics of the blockchain and the supply chain determine that the supply chain will be the hotspot of the blockchain. However, as a distributed ledger technology, the blockchain still has $51 \%$ of hidden dangers that trust between subjects remains risky. Starting from the perspective of computer technology, blockchain is designed with a set of algorithm with the mechanism of decentralized authority control of transactions, records and verification of real right transfer information, which will further facilitate supply chain control and traceability [8]. Based on blockchain technology, a highly integrated autonomous alliance information system capable of connecting supply chain alliances, financial institutions and government regulatory agencies can adjust the blockchain consensus mechanism and the inherent model of smart contracts to establish a random basis dynamic equity proof consensus mechanism (SDPoS)for supply chain alliances. Although there are many studies on the blockchain technology architecture, how to build "double-chain fusion" development technology architecture to avoid $51 \%$ of the attack problem remains to be deepened.

\section{Practical application research of "double chain" fusion}

At present, the blockchain has gradually changed from concept propaganda a few years ago to a focus on the service entity economy. In 2018, it was called the blockchain technology in the industry, and the world's major companies have tried the water blockchain field. The corresponding pilot projects cover six major fields of finance, logistics and food, including more than 100 top companies such as Ali, Tencent, Baidu, Huawei and Goldman Sachs[9-10].

In the logistics supply chain, maritime giant Maersk and IBM form a joint venture to jointly launch a blockchain crossborder supply chain project to help shippers, ports, customs, banks and other suppliers in the supply chain track shipment information. The tamper-proof digital recording method replaces paper files, which will further improve global trade efficiency. Companies such as American Express UPS, FedEx, and BNSF Railroad join the Global Blockchain Freight Alliance (BiTA), which aims to aggregate freight companies to develop a standard framework while testing blockchain applications. The modern logistics company (HMM) of the Korean logistics company uses the IoT equipment to monitor the temperature blockchain system of the refrigerated container in real time, which makes the arrival/departure, bill of lading and cargo tracking of the vessel adopt the "paperless" operation mode. As mentioned, blockchain technology will further enhance the operational efficiency of the logistics supply chain.

Many well-known companies are involved in food traceability. Companies such as Wal-Mart, Kroger, Nestle and Unilever have partnered with IBM to improve the supply chain tracking through blockchain, improve food safety and shorten the time to track mango shipments (from 7 days to 2.2 seconds); Alibaba's Tmall International launched a global traceability program to track commodity information using big data and blockchain; retail giant Carrefour deployed a super-book based blockchain food traceability in the Spanish network platform.
In summary, the food industry can achieve more efficient operation in safety recall with the support of blockchain technology.

\section{CONCLUSIONS}

At present, the research results of the development of blockchain and supply chain "double chain integration" mainly focus on supply chain information management, chain user credit management, product traceability and so on. Blockchain technology relies on its distributed ledger technology to ensure full sharing of information across the supply chain. A consensus mechanism is adopted to achieve a safe, effective and democratic consensus among the nodes. Relying on cryptography to build a strict identity authentication system and permission privacy system to restrict account access rights to ensure that data cannot be tampered and maintain the privacy of traders. Through the time stamp mechanism, data continuity can be guaranteed to facilitate data traceability. The intelligent contract mechanism can ensure that the pre-agreed trigger conditions are automatically executed when they are satisfied, thus effectively avoiding the revenue behavior. In short, the blockchain, with its unique technical characteristics, provides a technical means of solving the supply chain credit problem without interference.

Since the supply chain involves multiple parties such as suppliers, manufacturers, distributors, retailers, etc., there may be different interests and cooperation between the parties. Although the advantages of the blockchain are obvious, there are certain disadvantages and limitations in applying it directly to the supply chain:

(1) While it can greatly improve the informationization level of the supply chain, on the one hand, the blockchain system still takes the risk of being attacked by 51\%; on the other hand, it will bring a series of costs such as facility construction, technology popularization and personnel training in a short time. And the transparency of information will also bring about a change in the interests of members on the chain.

(2) Blockchain data is transparent, and you need to consider what data will be placed on the chain, and how information about personal sensitive information or trade secrets should be handled.

(3) When the physical product data is uploaded to the blockchain through the Internet of Things technology, in the blockchain, in order to ensure the smooth flow of information, how to add digital tags to achieve the purpose of tracking the physical products still requires technical solutions.

\section{RESEARCH PROSPECTS}

As the public is increasingly concerned about food and drug safety, care about the true value of products, and resist counterfeit and shoddy products, as companies continue to test in the supply chain, although the application of blockchain in the supply chain is still in its infancy, related research is deepening.

Although the concept of supply chain is prevalent in today's society, there are still many problems in supply chain management. According to the current development status of 
the supply chain and the industry development report, the future development trends of the supply chain mainly include: data visualization, process optimization and demand management, and product traceability. Using the latest achievements in various disciplines such as management, finance, statistics, and computer science to rationally allocate resources and optimize processes in the supply chain, and improve the efficiency and safety of the supply chain are one of the hotspots in the industry today. The development of

"double-chain convergence" can solve the problem of crossintegration and allocation of resources among different industrial chains in parallel. The main driving forces are as follows:

(1) Information transparency of blockchain can improve the overall efficiency of the upstream and downstream of the supply chain.

(2) The blockchain technology is not tampering and transparent, which reduces the difficulty of supervision.

(3) The blockchain's advantage of tracking counterfeit and shoddy goods caters to the needs of consumers.

(4) The development of information technology such as Internet of Things technology, big data, cloud computing, etc. further promotes the development of blockchain technology.

(5) Supply chain finance is a practical solution for a blockchain.

Combining the technical characteristics of blockchain decentralization and trustless with mature Internet of Things technology, big data, and cloud computing to build a supply chain information system, the digital platform has created a new environment for supply chain governance in the Internet. Through the construction of the entire chain of information technology in the supply chain, it can alleviate supply chain risks such as opportunism caused by information asymmetry, and further improve the level of intelligent management of supply chain, thus promoting the transformation of traditional supply chain to intelligent supply chain.

\section{REFERENCES}

[1] Nakamoto S. Bitcoin: A peer- to- peer electronic cash system[EB/OL].[2016-11-30]. https://bitcoin.org/bitcoin.pdf

[2] Jin Kai, Yang Ruizhe, Yang Zhaoxin, Lin Bo, Zhang Yanhua.Application of Blockchain in Supply Chain Management[J].Information Engineering, 2018, 4(03):29-38.(In Chinese)

[3] ZHU Jianming, FU Yonggui. Dynamic multi-center collaborative authentication model for supply chain based on blockchain[J]. Journal of Network and Information Security, 2016, 2(01): 27-33.(In Chinese)

[4] Wang Chuanlei, Wan Yiwei, Qin Qin, Wang Ningning. A Supply Chain Logistics Information Ecology Circle Model Based on Blockchain[J].Information Studies Theory \& Practice, 2017, 40(07):115121.(In Chinese)

[5] Yu Lina, Zhang Guofeng, Jia Jingdun, Gao Wanlin, Zhang Ganghong, Tao Sha. Modern Agricultural Product Supply Chain Based on Blockchain Technology[J]. Transactions of the Chinese Society of Agricultural Machinery, 2017, 48(S1): 387-393.(In Chinese)

[6] Tian F. [IEEE 2016 13th International Conference on Service Systems and Service Management (ICSSSM) - Kunming, China (2016.6.242016.6.26)] 2016 13th International Conference on Service Systems and Service Management (ICSSSM) - An agri-food supply chain traceability system for China based on RFID \& blockchain technology[C]// 2016 13th International Conference on Service Systems and Service Management (ICSSSM). IEEE, 2016:1-6.

[7] Reijers W, Coeckelbergh M . The Blockchain as a Narrative Technology: Investigating the Social Ontology and Normative Configurations of Cryptocurrencies[J]. Philosophy \& Technology, 2016, 31(1):1-28.

[8] Yang Huiqin, Sun Lei, Zhao Xichao. Construction of mutual trust and win-win supply chain information platform based on blockchain technology[J].Science \& Technology Progress and Policy, 2018, 35(05):21-31.(In Chinese)

[9] Zhao Shumei, Men Ruixue. "New Logistics" under the Background of "New Retail" [J]. China's Circulation Economy, 2019, 33 (03): 40-49.(In Chinese)

[10] Chen Zhongxian, Li Qinwei.Design of blockchain structure applied to supply chain[J].Electronic Technology and Software Engineering, 2019(05): 172-173.(In Chinese) 